\title{
Convergence of a Two-Step Iterative Method for Nondifferentiable Operators in Banach Spaces
}

\author{
Abhimanyu Kumar, ${ }^{1}$ Dharmendra K. Gupta $\mathbb{D}^{1},{ }^{1}$ Eulalia Martínez $\mathbb{D}^{\circ},{ }^{2}$ and Sukhjit Singh ${ }^{3}$ \\ ${ }^{1}$ Department of Mathematics, Indian Institute of Technology Kharagpur, Kharagpur 721302, India \\ ${ }^{2}$ Instituto Universitario de Matemática Multidisciplinar, Universitat Politècnica de València, Valencia, Spain \\ ${ }^{3}$ Department of Mathematical Science, Punjab Technical University Jalandhar, Jalandhar, India
}

Correspondence should be addressed to Eulalia Martínez; eumarti@mat.upv.es

Received 6 February 2018; Accepted 29 March 2018; Published 7 May 2018

Academic Editor: Danilo Comminiello

Copyright (C) 2018 Abhimanyu Kumar et al. This is an open access article distributed under the Creative Commons Attribution License, which permits unrestricted use, distribution, and reproduction in any medium, provided the original work is properly cited.

\begin{abstract}
The semilocal and local convergence analyses of a two-step iterative method for nonlinear nondifferentiable operators are described in Banach spaces. The recurrence relations are derived under weaker conditions on the operator. For semilocal convergence, the domain of the parameters is obtained to ensure guaranteed convergence under suitable initial approximations. The applicability of local convergence is extended as the differentiability condition on the involved operator is avoided. The region of accessibility and a way to enlarge the convergence domain are provided. Theorems are given for the existence-uniqueness balls enclosing the unique solution. Finally, some numerical examples including nonlinear Hammerstein type integral equations are worked out to validate the theoretical results.
\end{abstract}

\section{Introduction}

Let $\rho^{*}$ be the unique solution of

$$
H(x)=0,
$$

where $H: \mathscr{D} \subseteq \mathscr{X} \rightarrow \mathscr{Y}$ is a continuous nonlinear operator defined on a nonempty convex subset $\mathscr{D}$ of a Banach $\mathscr{X}$ with values in a Banach space $\mathcal{Y}$. This problem frequently occurs in numerical analysis. Many scientific and real-life problems can be formulated mathematically in terms of integral equations, boundary value problems, equilibrium theory, optimization, and differential equations whose solutions require solving (1). The solutions of discrete dynamical systems also require solving them in order to represent the equilibrium states of these systems. With the existence of high-speed computational devices which solve them faster and with more accuracy, the problem for solving nonlinear equations has further gained and added advantages. Generally, iterative methods along with their convergence analysis are used to find the solutions of these equations. Many researchers [1-6] have extensively studied these problems and proposed many direct and iterative methods for their solutions along with their semilocal $[1-3,7]$, local $[8,9]$, and global convergence analysis [10]. In semilocal convergence analysis, we impose conditions on starting points while local convergence analysis requires the condition on the solution. Global study of convergence generally depends on the type of operators involved. The well-known quadratically convergent Newton's method [11, 12] used for (1) is given by

$$
x_{k+1}=x_{k}-\Gamma_{k} H\left(x_{k}\right), \quad k \geq 0,
$$

where $x_{0} \in \mathscr{D}$ and $\Gamma_{k}=H^{\prime}\left(x_{k}\right)^{-1} \in L(\mathscr{Y}, \mathscr{X})$. Here, $L(\mathscr{Y}, \mathscr{X})$ denotes the set of bounded linear operators from $\mathscr{Y}$ into $\mathscr{X}$. In [13], a family representing third-order iterative methods for (1) is given for $k \geq 0$ by

$$
\begin{gathered}
x_{\alpha, k+1}=x_{\alpha, k}-\left[I+\frac{1}{2} G_{H}\left(x_{\alpha, k}\right)\left[I-\alpha G_{H}\left(x_{\alpha, k}\right)\right]^{-1}\right] \\
\cdot H^{\prime}\left(x_{\alpha, k}\right)^{-1} H\left(x_{\alpha, k}\right),
\end{gathered}
$$

where $x_{\alpha, 0}$ is the starting iterate and $G_{H}(x)=$ $H^{\prime}(x)^{-1} H^{\prime \prime}(x) H^{\prime}(x)^{-1} H(x)$. This family contains the Chebyshev method $(\alpha=0)$, the Halley method $(\alpha=1 / 2)$, 
and the Super-Halley method $(\alpha=1)$, respectively. These methods and many others use the differentiability of $H$. Not much work is done by using nondifferentiability of $H$ which can be expressed in the form

$$
H(x)=F(x)+G(x)=0,
$$

where $F, G: \mathscr{D} \subseteq \mathscr{X} \rightarrow \mathcal{Y}$ are continuous and nonlinear operators. $F$ is differentiable while $G$ is continuous only and not differentiable. In [14], a quadratic order iterative method for (4) is given by

$$
\begin{aligned}
& x_{k+1}= x_{k} \\
&-\left(F^{\prime}\left(x_{k}\right)+\left[x_{k-1}, 2 x_{k}-x_{k-1} ; G\right]\right)^{-1} H\left(x_{k}\right), \\
& k \geq 0,
\end{aligned}
$$

where $x_{-1}$ and $x_{0}$ are two starting points and $[x, y ; G] \epsilon$ $L(\mathscr{X}, \mathcal{Y})$ satisfying $[x, y ; G](x-y)=G(x)-G(y)$ for $x, y \in \mathscr{D}$ and $x \neq y$.

Consider the following two-step difference differential method [15] for solving (4) given by

$$
\begin{aligned}
& x_{k+1}=x_{k}-A_{k}^{-1} H\left(x_{k}\right), \\
& y_{k+1}=x_{k+1}-A_{k}^{-1} H\left(x_{k+1}\right), \quad k \geq 0,
\end{aligned}
$$

where $x_{0}, y_{0} \in \mathscr{D}$ are two starting points and $A_{k}=\left[F^{\prime}\left(\left(x_{k}+\right.\right.\right.$ $\left.\left.\left.y_{k}\right) / 2\right)+\left[x_{k}, y_{k} ; G\right]\right]$. Its local convergence analysis with super quadratic order is described. The differentiability condition on $H^{\prime}\left(\rho^{*}\right)$ which restricts the applicability of (6) is also used. Moreover, no numerical examples were worked out. Recently, some special cases of (6) are also studied. One special case of $(6)$ is given in $[1,16]$ for $F=0$. Another one is given in [17] for $G=0$. The importance of (6) lies in the fact that it uses the memory of $H\left(x_{k}\right)$ in each iterate. If we consider the evaluation of $H\left(x_{k}\right)$ as one function evaluation, then the total number of function evaluations of (6) for solving (4) coincides with that given by (5). However, the rate of convergence of (6) is much faster. This is shown by the following example.

Example 1. Consider

$$
\begin{gathered}
u^{3 / 2}-v-\frac{3}{4}+\frac{1}{9}|u-1|=0 \\
v^{3 / 2}+\frac{2}{9} u-\frac{3}{8}+\frac{1}{9}|v|=0,
\end{gathered}
$$

where $x=(u, v) \in \mathbb{R}^{2}$.

Take $F(u, v)=\left(\begin{array}{l}F_{1}(u, v) \\ F_{2}(u, v)\end{array}\right)=\left(\begin{array}{c}u^{3 / 2}-v-3 / 4 \\ v^{3 / 2}+(2 / 9) u-3 / 8\end{array}\right)$ and $G(u, v)=$ $\left(\begin{array}{l}G_{1}(u, v) \\ G_{2}(u, v)\end{array}\right)=\left(\begin{array}{c}(1 / 9)|u-1| \\ (1 / 9)|v|\end{array}\right)$.

A comparison of the absolute error approximation obtained by (6) and (5) with tolerance $\left\|\rho^{*}-x_{k}\right\|<10^{-20}$ is given in Table 1 .

In this study, the semilocal and local convergence analyses of a two-step iterative method for nonlinear nondifferentiable operators are described in Banach spaces. The recurrence relations are derived under weaker conditions on the operator. For semilocal convergence, the domain of parameters is obtained to ensure guaranteed convergence under suitable initial approximations. The applicability of local convergence is extended as the differentiability condition on the involved operator is avoided. The region of accessibility and a way to enlarge the convergence domain are provided. Theorems are given for the existence-uniqueness balls enclosing the unique solution. Finally, some numerical examples including nonlinear Hammerstein type integral equations are worked out to validate the theoretical results.

The paper is organized as follows. The Introduction forms Section 1. In Section 2, semilocal convergence of (6) is established. In Section 2.1, some special cases and domain of parameters are given to ensure the initial points for guaranteed convergence of (6). In Section 3, local convergence analysis of (6) is established. Some special cases and region of accessibility are also discussed. In Section 4, some numerical examples including nonlinear Hammerstein type integral equations are given to validate the theoretical results. Finally, conclusions are given in Section 5.

\section{Semilocal Convergence}

This section describes the semilocal convergence of (6) for solving (1). Let $\mathscr{B}(x, r)$ and $\overline{\mathscr{B}}(x, r)$ denote the open and closed balls with center at $x$ and radius $r$, respectively. Let $x_{0}$ and $y_{0}$ be suitably chosen initial approximations and $\alpha>0$, $\beta>0, \eta>0$, and $\zeta>0$ be some positive real numbers. Define a class $S\left(\alpha, \beta, \eta, \zeta, \omega_{1}, \omega_{2}\right)$, where $\omega_{1}$ and $\omega_{2}$ are to be defined. The triplet $\left(H, x_{0}, y_{0}\right) \in S$ if

$\left(C_{1}\right)\left\|x_{0}-y_{0}\right\| \leq \alpha$ for $x_{0}, y_{0} \in \mathscr{D}$;

$\left(C_{2}\right) A_{0}^{-1} \in L(\mathcal{Y}, \mathscr{X})$ such that $\left\|A_{0}^{-1}\right\| \leq \beta$ and $\left\|H\left(x_{0}\right)\right\| \leq \zeta$;

$\left(C_{3}\right)\left\|A_{0}^{-1} H\left(x_{0}\right)\right\| \leq \beta \zeta=\eta$;

$\left(C_{4}\right)\left\|A_{0}^{-1}\left(F^{\prime}(x)-F^{\prime}(y)\right)\right\| \leq \omega_{1}(\|x-y\|)$, where $\omega_{1}: \mathbb{R}_{+} \rightarrow$ $\mathbb{R}_{+}$is a continuous and nondecreasing function for $x, y \in \mathscr{D}$;

$\left(C_{5}\right)\left\|A_{0}^{-1}([x, y ; G]-[u, v ; G])\right\| \leq \omega_{2}(\|x-u\|, \| y-$ $v \|)$, where $\omega_{2}: \mathbb{R}_{+} \times \mathbb{R}_{+} \rightarrow \mathbb{R}_{+}$is a continuous and nondecreasing function in its both arguments for $x, y, u, v \in \mathscr{D}$;

$\left(C_{6}\right)$ the equation

$$
\eta+\operatorname{tg}(t)-t=0
$$

where $g(t)=M /\left(1-\left(\omega_{1}((2 t+\alpha) / 2)+\omega_{2}(t, t+\alpha)\right)\right)$, $M=\max \left(M_{1}, M_{2}\right), M_{1}=\int_{0}^{1} \omega_{1}((\alpha+2 \eta \gamma) / 2) d \gamma+$ $\omega_{2}(\eta, \alpha)$, and $M_{2}=\int_{0}^{1} \omega_{1}((\eta+2 \eta \gamma) / 2) d \gamma+\omega_{2}(\eta, \eta)$, has at least one positive root. The smallest positive root is denoted by $R$;

$\left(C_{7}\right) \mathscr{B}\left(x_{0}, R\right) \subseteq \mathscr{D}$;

$\left(C_{8}\right) M+\widetilde{M}<1$, where $\widetilde{M}=\omega_{1}((2 R+\alpha) / 2)+\omega_{2}(R, R+\alpha)$. 
TABLE 1: Comparison of absolute error.

\begin{tabular}{lcc}
\hline$k$ & Method $(6)$ & Method (5) \\
\hline 1 & $\left\|\rho^{*}-x_{k}\right\|,\left(x_{0}, y_{0}\right)=((2,2),(1,0))$ & $\left\|\rho^{*}-x_{k}\right\|=\left(x_{-1}, x_{0}\right)=((2,2),(1,0))$ \\
2 & $6.83883 \ldots \times 10^{-02}$ & $6.66148 \times 10^{-02}$ \\
3 & $2.29390 \ldots \times 10^{-04}$ & $2.15254 \times 10^{-03}$ \\
4 & $2.28203 \ldots \times 10^{-10}$ & $5.65881 \times 10^{-06}$ \\
5 & $3.26673 \ldots \times 10^{-18}$ & $3.36400 \times 10^{-07}$ \\
6 & & $1.99969 \times 10^{-08}$ \\
7 & & $1.18869 \times 10^{-09}$ \\
8 & & $7.06605 \times 10^{-11}$ \\
9 & & $4.20033 \times 10^{-12}$ \\
10 & & $2.49683 \times 10^{-13}$ \\
\hline
\end{tabular}

Lemma 2. For operators $F, G$, and $H$ defined in (4) and (5), it follows that, for all $k \geq 0$,

$$
\begin{aligned}
& H\left(x_{k+1}\right)=\int_{0}^{1}\left(F^{\prime}\left(x_{k}+t\left(x_{k+1}-x_{k}\right)\right)\right. \\
& \left.\quad-F^{\prime}\left(\frac{x_{k}+y_{k}}{2}\right)\right) d t\left(x_{k+1}-x_{k}\right) \\
& \quad+\left(\left[x_{k+1}, x_{k} ; G\right]-\left[x_{k}, y_{k} ; G\right]\right)\left(x_{k+1}-x_{k}\right) .
\end{aligned}
$$

Proof. The proof is simple and hence omitted here.

Lemma 3. Under conditions $\left(C_{1}\right)-\left(C_{8}\right)$ and parameters introduced in Section 2, we have that if $\left(H, x_{0}, y_{0}\right) \in S$, then the following recurrence relations hold for $k \geq 1$ :

(i) There exists $A_{k}^{-1}=\left(F^{\prime}\left(\left(x_{k}+y_{k}\right) / 2\right)+\left[x_{k}, y_{k} ; G\right]\right)^{-1}$ such that $\left\|A_{k}^{-1} A_{0}\right\| \leq 1 /(1-\widetilde{M})$.

(ii) $\left\|x_{k+1}-x_{k}\right\| \leq g(R)\left\|x_{k}-x_{k-1}\right\|$.

(iii) $\left\|y_{k}-x_{k}\right\| \leq g(R)\left\|x_{k}-x_{k-1}\right\|$.

(iv) $\left\|x_{k+1}-x_{0}\right\| \leq \sum_{i=0}^{i=k} g(R)^{i}\left\|x_{1}-x_{0}\right\|<R$.

(v) $\left\|y_{k}-x_{0}\right\| \leq \sum_{i=0}^{i=k} g(R)^{i}\left\|x_{1}-x_{0}\right\|<R$.

Proof. This lemma can be proved by mathematical induction. Clearly, we have $\left\|x_{1}-x_{0}\right\| \leq \eta<R$, and using Lemma 2, we get

$$
\begin{aligned}
& \left\|y_{1}-x_{1}\right\|=\left\|A_{0}^{-1} H\left(x_{1}\right)\right\| \\
& \quad=\| A_{0}^{-1}\left(\int_{0}^{1}\left(F^{\prime}\left(x_{0}+t\left(x_{1}-x_{0}\right)\right)-F^{\prime}\left(\frac{x_{0}+y_{0}}{2}\right)\right) d t\right. \\
& \left.\quad+\left(\left[x_{1}, x_{0} ; G\right]-\left[x_{0}, y_{0} ; G\right]\right)\right)\left(x_{1}-x_{0}\right) \| \\
& \quad \leq\left(\int_{0}^{1} \omega_{1}\left(\frac{\left\|x_{0}-y_{0}\right\|+2 t\left\|x_{1}-x_{0}\right\|}{2}\right) d t\right. \\
& \left.\quad+\omega_{2}\left(\left\|x_{1}-x_{0}\right\|,\left\|x_{0}-y_{0}\right\|\right)\right)\left\|x_{1}-x_{0}\right\|
\end{aligned}
$$

$$
\begin{aligned}
& \leq\left(\int_{0}^{1} \omega_{1}\left(\frac{\alpha+2 t \eta}{2}\right) d t+\omega_{2}(\eta, \alpha)\right)\left\|x_{1}-x_{0}\right\| \\
& \leq M\left\|x_{1}-x_{0}\right\| \leq g(R)\left\|x_{1}-x_{0}\right\| .
\end{aligned}
$$

Now,

$$
\begin{aligned}
\left\|y_{1}-x_{0}\right\| & \leq\left\|y_{1}-x_{1}\right\|+\left\|x_{1}-x_{0}\right\| \\
& \leq(1+g(R))\left\|x_{1}-x_{0}\right\|<R .
\end{aligned}
$$

Thus, $x_{1}, y_{1} \in \mathscr{B}\left(x_{0}, R\right)$. Let us assume that the induction is true for some $k \leq r$. Then,

$$
\left\|\frac{x_{k}+y_{k}}{2}-x_{0}\right\| \leq \frac{\left\|x_{k}-x_{0}\right\|+\left\|y_{k}-x_{0}\right\|}{2} \leq R .
$$

This gives $x_{k}, y_{k},\left(x_{k}+y_{k}\right) / 2 \in \mathscr{B}\left(x_{0}, R\right)$. Now,

$$
\begin{aligned}
\| I- & A_{0}^{-1} A_{k}\|=\| A_{0}^{-1}\left(A_{k}-A_{0}\right) \| \\
\leq & \left\|A_{0}^{-1}\left(F^{\prime}\left(\frac{x_{k}+y_{k}}{2}\right)-F^{\prime}\left(\frac{x_{0}+y_{0}}{2}\right)\right)\right\| \\
& +\left\|A_{0}^{-1}\left(\left[x_{k}, y_{k} ; G\right]-\left[x_{0}, y_{0} ; G\right]\right)\right\| \\
\leq & \omega_{1}\left(\frac{\left\|x_{k}-x_{0}\right\|+\left\|y_{k}-x_{0}\right\|+\left\|x_{0}-y_{0}\right\|}{2}\right) \\
& +\omega_{2}\left(\left\|x_{k}-x_{0}\right\|,\left\|y_{k}-x_{0}\right\|+\left\|x_{0}-y_{0}\right\|\right) \\
\leq & \omega_{1}\left(\frac{2 R+\alpha}{2}\right)+\omega_{2}(R, R+\alpha)<1 .
\end{aligned}
$$

Using Banach Lemma on invertible operators [4], we get

$$
\left\|A_{k}^{-1} A_{0}\right\| \leq \frac{1}{1-\widetilde{M}}
$$


Using Lemma 2 and (6), we get

$$
\begin{aligned}
& \left\|x_{k+1}-x_{k}\right\| \leq\left\|A_{k}^{-1} A_{0}\right\|\left\|A_{0}^{-1} H\left(x_{k}\right)\right\| \\
& \quad=\frac{1}{1-\widetilde{M}}\left(\| A_{0}^{-1}\left(F^{\prime}\left(x_{k-1}+t\left(x_{k}-x_{k-1}\right)\right)\right.\right. \\
& \left.\quad-F^{\prime}\left(\frac{x_{k-1}+y_{k-1}}{2}\right)\right)\|+\| A_{0}^{-1}\left(\left[x_{k}, x_{k-1} ; G\right]\right. \\
& \left.\left.\quad-\left[x_{k-1}, y_{k-1} ; G\right]\right) \|\right)\left\|x_{k}-x_{k-1}\right\| \\
& \quad \leq \frac{1}{1-\widetilde{M}}\left[\int_{0}^{1} \omega_{1}\left(\frac{\left\|x_{k-1}-y_{k-1}\right\|+2 t\left\|x_{k}-x_{k-1}\right\|}{2}\right) d t\right. \\
& \left.\quad+\omega_{2}\left(\left\|x_{k}-x_{k-1}\right\|,\left\|x_{k-1}-y_{k-1}\right\|\right)\right]\left\|x_{k}-x_{k-1}\right\| \leq g(R) \\
& \quad \cdot\left\|x_{k}-x_{k-1}\right\| .
\end{aligned}
$$

Following in a similar manner, it can be derived that

$$
\left\|y_{k}-x_{k}\right\| \leq g(R)\left\|x_{k}-x_{k-1}\right\|
$$

This implies (i)-(iii). (iv) and (v) can easily be derived using $S$ and (i)-(iii) recursively. Thus, the lemma is proved.

Theorem 4. Let $H: \mathscr{D} \subset \mathscr{X} \rightarrow \mathscr{Y}$ verify conditions $\left(C_{1}\right)-\left(C_{8}\right)$ such that $\left(H, x_{0}, y_{0}\right) \in S$. Then, starting with $x_{0}, y_{0}$, the sequences $\left\{x_{k}\right\}$ and $\left\{y_{k}\right\}$ generated by (6) are well defined, remain in $\mathscr{B}\left(x_{0}, R\right)$, and converge to the solution $\rho^{*}$ of $(1)$. Moreover, $\rho^{*}$ is unique in $\overline{\mathscr{B}}\left(x_{0}, R\right)$.

Proof. From Lemmas 2 and 3 , it is clear that $x_{k}$ and $y_{k}$ are well defined and belong to $\mathscr{B}\left(x_{0}, R\right)$. First, we shall show that $\left\{x_{k}\right\}$ is a cauchy sequence. For fixed $k$ and $m \geq 1$, we get

$$
\begin{aligned}
& \left\|x_{k+m}-x_{k}\right\| \leq\left\|x_{k+m}-x_{k+m-1}\right\|+\cdots+\left\|x_{k+1}-x_{k}\right\| \\
& \leq\left(g(R)^{m-1}+g(R)^{m-2}+\cdots+g(R)+1\right) \\
& \cdot\left\|x_{k+1}-x_{k}\right\| \\
& \leq\left(g(R)^{m-1}+g(R)^{m-2}+\cdots+g(R)+1\right) \\
& \cdot\left\|x_{k+1}-x_{k}\right\| \leq\left(\frac{1-g(R)^{m}}{1-g(R)}\right) g(R)^{k}\left\|x_{1}-x_{0}\right\| .
\end{aligned}
$$

This gives $x_{k} \rightarrow \rho^{*}$ as $k \rightarrow \infty$. Now, to show that $\rho^{*}$ is a solution of (1), we have

$$
\left\|A_{0}^{-1} H\left(x_{k}\right)\right\| \leq M\left\|x_{k}-x_{k-1}\right\|
$$

and $\left\|x_{k}-x_{k-1}\right\| \rightarrow 0$ as $k \rightarrow \infty$. This gives $H\left(\rho^{*}\right)=0$. Next, to show the uniqueness of $\rho^{*}$, let us assume that $\sigma^{*}$ is another solution of $(1)$ in $\overline{\mathscr{B}}\left(x_{0}, R\right)$. For $P=\int_{0}^{1} F^{\prime}\left(\rho^{*}+t\left(\sigma^{*}-\rho^{*}\right)\right) d t+$ $\left[\rho^{*}, \sigma^{*} ; G\right]$, we get

$$
\begin{aligned}
\| I & -A_{0}^{-1} P\|\leq\| A_{0}^{-1}\left(A_{0}-P\right) \| \\
& \leq \| A_{0}^{-1}\left(\int_{0}^{1} F^{\prime}\left(\rho^{*}+t\left(\sigma^{*}-\rho^{*}\right)\right) d t\right. \\
& \left.-F^{\prime}\left(\frac{x_{0}+y_{0}}{2}\right)\right)\|+\| A_{0}^{-1}\left(\left[\rho^{*}, \sigma^{*} ; G\right]\right. \\
& \left.-\left[x_{0}, y_{0} ; G\right]\right) \| \leq \int_{0}^{1} \omega_{1}\left(\|(1-t)\left(\rho^{*}-x_{0}\right)\right. \\
& \left.+t\left(\sigma^{*}-x_{0}\right)+x_{0}-\frac{x_{0}+y_{0}}{2} \|\right)+\omega_{2}\left(\left\|\rho^{*}-x_{0}\right\|,\right. \\
& \left.\left\|\sigma^{*}-x_{0}\right\|+\left\|x_{0}-y_{0}\right\|\right) \leq \omega_{1}\left(\frac{2 R+\alpha}{2}\right)+\omega_{2}(R, R \\
& +\alpha)=\widetilde{M}<1 .
\end{aligned}
$$

Using Banach Lemma on invertible operators, we get that $P^{-1}$ exists and $\left\|P^{-1} A_{0}\right\| \leq 1 /(1-\widetilde{M})$. Taking norm on both sides on $P\left(\sigma^{*}-\rho^{*}\right)=H\left(\sigma^{*}\right)-H\left(\rho^{*}\right)$, we get that $\sigma^{*}=\rho^{*}$. This shows the uniqueness and thus the theorem is proved.

2.1. Some Special Cases and Domain of Parameters. In this subsection, some special cases of Theorem 4 and the iterative method (6) are presented. We find the domain of parameters to get the set of initial approximations for the guaranteed convergence of (6) for $F=0$. Consider $G: \mathbb{R}^{n} \rightarrow \mathbb{R}^{n}$, given by

$$
G(t)=t-g-T\left(\xi_{1} v_{t}+\xi_{2} w_{t}\right)=0,
$$

where $g$ is a nonlinear vector function of size $n \times 1, T$ is a matrix of size $n \times n, t=\left(t_{1}, t_{2}, \ldots, t_{n}\right)^{T}, v_{t}=$ $\left(t_{1}^{2}, t_{2}^{2}, \ldots, t_{n}^{2}\right)^{T}, w_{t}=\left(\left|t_{1}\right|,\left|t_{2}\right|, \ldots,\left|t_{n}\right|\right)^{T}$, and $\xi_{1}, \xi_{2} \in \mathbb{R}-\{0\}$. It can be observed that

$$
\begin{array}{r}
\|[x, y ; G]-[u, v ; G]\| \leq K_{1}+K_{2}(\|x-u\|+\|y-v\|), \\
x, y, u, v \in \mathbb{R}^{n}
\end{array}
$$

with $K_{1}=2\left|\xi_{2}\right|\|T\|$ and $K_{2}=\left|\xi_{1}\right|\|T\|$. Earlier studies $[1,15,16]$ for nondifferentiable operators do not satisfy this condition. For $K_{1}=0$, this condition holds for differentiable operators. Now, we can take $\omega_{2}(\|x-u\|,\|y-v\|)=K_{1}+K_{2}(\|x-u\|+\|y-v\|)$ as a special case of condition $\left(C_{5}\right)$ of class $S$.

Theorem 5. Let $G: \mathscr{D} \subset \mathscr{X} \rightarrow \mathscr{Y}$ and $R$ be the smallest positive real number satisfying

$$
\left(1+\frac{T_{1}}{1-T_{2}-T_{3}(t)}\right) \eta-t=0,
$$

where $T_{1}=\beta\left(K_{1}+K_{2}(\eta+\alpha)\right), T_{2}=\beta\left(K_{1}+2 K_{2} \eta\right)$, and $T_{3}(t)=$ $\beta\left(K_{1}+K_{2}(2 t+\alpha)\right)$. Suppose that $T_{1}+T_{3}(R)<1, T_{2}+T_{3}(R)<1$, and $\mathscr{B}\left(x_{0}, R\right) \subseteq \mathscr{D}$. Starting with $x_{0}$ and $y_{0}$, the sequences $\left\{x_{k}\right\}$ and $\left\{y_{k}\right\}$ generated by (6) are well defined and converge to $\rho^{*}$ of $G(x)=0$. Moreover, $\rho^{*}$ is unique in $\overline{\mathscr{B}}\left(x_{0}, R\right)$. 
Proof. From $S$, we get $\left\|x_{1}-x_{0}\right\| \leq \eta<R$. Take $D_{k}=$ $\left[x_{k}, y_{k} ; G\right]$. The proof is given by mathematical induction. Let $x_{k}, y_{k}$, and $\left(x_{k}+y_{k}\right) / 2 \in \mathscr{B}\left(x_{0}, R\right)$ for $k \geq 1$. Now,

$$
\begin{aligned}
\| I & -D_{0}^{-1} D_{k}\|\leq\| D_{0}^{-1} \|\left(\left\|\left[x_{k}, y_{k} ; G\right]-\left[x_{0}, y_{0} ; G\right]\right\|\right) \\
& \leq \beta\left(K_{1}+K_{2}\left(\left\|x_{k}-x_{0}\right\|+\left\|y_{k}-x_{0}\right\|+\left\|x_{0}-y_{0}\right\|\right)\right) \\
& \leq \beta\left(K_{1}+K_{2}(2 R+\alpha)\right)=T_{3}(R) .
\end{aligned}
$$

Using Banach Lemma, it is given that

$$
\left\|D_{k}^{-1}\right\| \leq \frac{\beta}{1-T_{3}(R)}
$$

Again,

$$
\begin{aligned}
& \left\|G\left(x_{k}\right)\right\|=\left\|G\left(x_{k-1}\right)-\left(G\left(x_{k-1}\right)-G\left(x_{k}\right)\right)\right\| \\
& \quad \leq\left\|\left[x_{k}, x_{k-1} ; G\right]-\left[x_{k-1}, y_{k-1} ; G\right]\right\|\left(\left\|x_{k}-x_{k-1}\right\|\right) \\
& \quad \leq\left[K_{1}+K_{2}\left(\left\|x_{k}-x_{k-1}\right\|+\left\|y_{k-1}-x_{k-1}\right\|\right)\right] \| x_{k} \\
& \quad-x_{k-1} \|, \\
& \left\|x_{k+1}-x_{k}\right\| \\
& \quad \leq \frac{\beta\left[K_{1}+K_{2}\left(\left\|x_{k}-x_{k-1}\right\|+\left\|y_{k-1}-x_{k-1}\right\|\right)\right]}{1-T_{3}(R)} \| x_{k} \\
& \quad-x_{k-1} \| .
\end{aligned}
$$

This gives

$$
\begin{aligned}
& \left\|y_{k+1}-x_{k+1}\right\| \\
& \quad \leq \frac{\beta\left[K_{1}+K_{2}\left(\left\|x_{k+1}-x_{k}\right\|+\left\|y_{k}-x_{k}\right\|\right)\right]}{1-T_{3}(R)} \| x_{k+1} \\
& \quad-x_{k} \|,
\end{aligned}
$$

$$
\begin{aligned}
& \left\|x_{k+1}-x_{0}\right\| \leq\left(1+\frac{T_{1}}{1-T_{3}(R)}\left(1+\frac{T_{2}}{1-T_{3}(R)}\right.\right. \\
& \left.\left.\quad+\left(\frac{T_{2}}{1-T_{3}(R)}\right)^{2}+\cdots+\left(\frac{T_{2}}{1-T_{3}(R)}\right)^{k-1}\right)\right) \eta \\
& \quad \leq\left(1+\frac{T_{1}}{1-T_{3}(R)}\left(\frac{1-\left(T_{2} /\left(1-T_{3}(R)\right)\right)^{k}}{1-\left(T_{2} /\left(1-T_{3}(R)\right)\right)}\right)\right) \eta \\
& \quad<R .
\end{aligned}
$$

This shows that $x_{k+1} \in \mathscr{B}\left(x_{0}, R\right)$. Similarly, we can have that $y_{k+1} \in \mathscr{B}\left(x_{0}, R\right)$. Now, we obtain that $\left\{x_{k}\right\}$ is a Cauchy sequence. For fixed $k$ and $m \geq 1$, we get

$$
\begin{gathered}
\left\|x_{k+m}-x_{k}\right\| \leq\left\|x_{k+m}-x_{k+m-1}\right\|+\cdots+\left\|x_{k+1}-x_{k}\right\| \\
\leq\left(\frac{T_{2}^{m-1}}{\left(1-T_{3}(R)\right)^{m-1}}+\cdots+\frac{T_{2}}{\left(1-T_{3}(R)\right)}+1\right) \\
\cdot\left\|x_{k+1}-x_{k}\right\| \leq\left(\frac{1-T_{2}^{m} /\left(1-T_{3}(R)\right)^{m}}{1-T_{2} /\left(1-T_{3}(R)\right)}\right) \\
\cdot\left\|x_{k+1}-x_{k}\right\| \leq\left(\frac{1-T_{2}^{m} /\left(1-T_{3}(R)\right)^{m}}{1-T_{2} /\left(1-T_{3}(R)\right)}\right) \\
\cdot \frac{T_{2}^{k-1} T_{1}}{\left(1-T_{3}(R)\right)^{k}} \eta .
\end{gathered}
$$

Thus, $x_{k} \rightarrow \rho^{*}$ as $k \rightarrow \infty$. Now, to show that $\rho^{*}$ is a solution of (4), we get

$$
\left\|G\left(x_{k}\right)\right\| \leq T\left\|x_{k}-x_{k-1}\right\|, \quad \text { where } T=\max \left(T_{1}, T_{2}\right)
$$

and $\left\|x_{k}-x_{k-1}\right\| \rightarrow 0$ as $k \rightarrow \infty$. This gives $G\left(\rho^{*}\right)=0$. Uniqueness of $\rho^{*}$ can be shown in a similar manner given in Theorem 4. This proves the theorem.

Now, we present the domain of the parameters associated with Theorem 5. The domain of the parameters represents the set of all those points in $x y$ plane that allow the guaranteed convergence of (6) from the initial conditions used in Theorem 5. Transform (22) into the quadratic equation

$$
a_{0} t^{2}+a_{1} t+a_{2}=0 \text {, where, } a_{0}=2 K_{2} \beta, a_{1}=2 K_{1} \beta+K_{2} \alpha \beta-1, a_{2}=-\eta\left(K_{1} \beta+K_{2} \beta \eta-1\right) \text {. }
$$

Following [2], it is easy to see that (29) has two positive real roots, if

$$
\text { i.e., } 2 K_{1} \beta+K_{2} \alpha \beta+\sqrt{8 K_{2} \eta\left(1-K_{1} \beta-K_{2} \beta \eta\right)}<1 \text {. }
$$

$$
a_{2}>0 \text {, }
$$

i.e., $\eta\left(1-K_{1} \beta-K_{2} \beta \eta\right)>0$,

$$
a_{1}+\sqrt{4 a_{0} a_{2}}<0,
$$

Using (30), it is necessary to take $2 K_{1} \beta+K_{2} \beta \eta<1$ for the existence of positive real roots. Moreover, the smallest positive real root is given by 


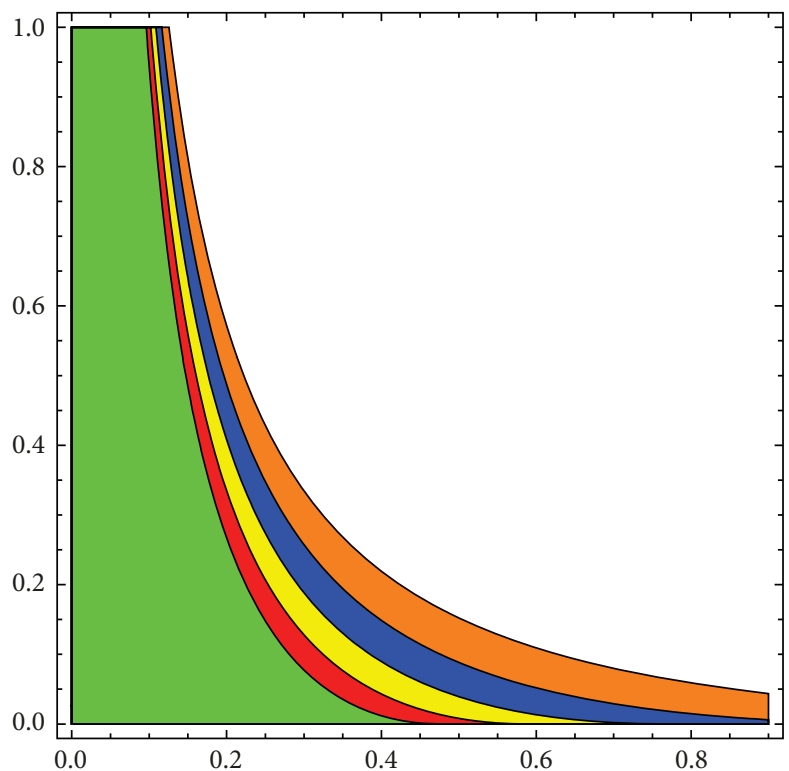

FIGURE 1: Domain of parameters of (6) for $Q=0.5$ and $K_{1}=0,0.2,0.4,0.6,0.8$ represented by orange, blue, yellow, red, and green colors, respectively.

$$
R_{1}=\frac{\left(1-2 K_{1} \beta-K_{2} \beta \eta\right)-\sqrt{\left(2 K_{1} \beta+K_{2} \alpha \beta-1\right)^{2}-8 K_{2} \beta \eta\left(1-K_{1} \beta-K_{2} \beta \eta\right)}}{4 K_{2} \beta} .
$$

Next, we take $\beta=x, K_{2} \eta=y$, and $Q=K_{2} \alpha$ and draw the domain of the parameters which gives the relation between some initial estimations. For this, we follow the criteria of Theorem 5 distinguished by two different cases. The first case is when $K_{1}=0$ (differentiable case) and the second case is when $K_{1} \neq 0$ (nondifferentiable case).

It can be seen in Figure 1 that, with the increasing value of $K_{1}$, we get a smaller region of the domain of parameters. By treating $K_{2}$ as a constant, it can be concluded here that the decrease of the value of $\alpha$ increases the domain of the parameter. This can be verified from Figure 2 .

\section{Local Convergence}

In this section, we shall establish the local convergence analysis of (6). Recently, this is given in [15] under the condition on $H^{\prime}\left(\rho^{*}\right)$, which is much more restrictive as it includes the differentiability of divided difference terms involved in (6). This restriction is removed and the following set of conditions is defined as class $U$. We say that the triplet $\left(H, \rho^{*}, \bar{\rho}\right) \in U$ if we have the following.

$\left(L_{1}\right)\left\|F^{\prime}(x)-F^{\prime}(y)\right\| \leq \sigma_{1}(\|x-y\|)$, where $\sigma_{1}(t)$ is a continuous and nondecreasing function defined on $\mathbb{R}_{+}$with values in $\mathbb{R}_{+}$for $x, y \in \mathscr{D}$.

$\left(L_{2}\right)\|[x, y ; G]-[u, v ; G]\| \leq \sigma_{2}(\|x-u\|,\|y-v\|)$, where $\sigma_{2}(r, s)$ is a continuous and nondecreasing function in its both arguments defined on $\mathbb{R}_{+} \times \mathbb{R}_{+}$with values in $\mathbb{R}_{+}$for $x, y, u, v \in \mathscr{D}$.

$\left(L_{3}\right)$ Let $\rho^{*}$ be such that $H\left(\rho^{*}\right)=0$. Take $\bar{\rho} \in \mathscr{D}$ and $\left\|\rho^{*}-\bar{\rho}\right\|=\theta>0$ so that the operator $A^{*}=F^{\prime}\left(\rho^{*}\right)+$ $\left[\rho^{*}, \bar{\rho} ; G\right]$ is invertible and $\left\|A^{*-1}\right\| \leq \lambda$.

$\left(L_{4}\right)$ The equation

$$
\begin{aligned}
& \lambda\left(\int_{0}^{1} \sigma_{1}((1+u) t) d u+\sigma_{2}(t, 2 t)+\sigma_{1}(t)\right. \\
& \left.\quad+\sigma_{2}(t, t+\theta)\right)-1=0
\end{aligned}
$$

has a positive real root. Let the minimum positive root be denoted by $R^{*}$.

$\left(L_{5}\right) \mathscr{B}\left(\rho^{*}, R^{*}\right) \subseteq \mathscr{D}$ and $\lambda\left(\sigma_{1}\left(R^{*}\right)+\sigma_{2}\left(R^{*}, R^{*}+\theta\right)\right)<1$.

Lemma 6. Let $\left(H, \rho^{*}, \bar{\rho}\right) \in$ L. If $x, y$, and $(x+y) / 2 \epsilon$ $\mathscr{B}\left(\rho^{*}, R^{*}\right) \subset \mathscr{D}$, then the operator $\left(F^{\prime}((x+y) / 2)+[x, y ; G]\right)$ is invertible and $\left\|\left(F^{\prime}((x+y) / 2)+[x, y ; G]\right)^{-1}\right\| \leq \lambda /\left(1-\left(\sigma_{1}\left(R^{*}\right)+\right.\right.$ $\left.\left.\sigma_{2}\left(R^{*}, R^{*}+\theta\right)\right)\right)$.

Proof. We prove this lemma using the definition of class $U$. We consider 


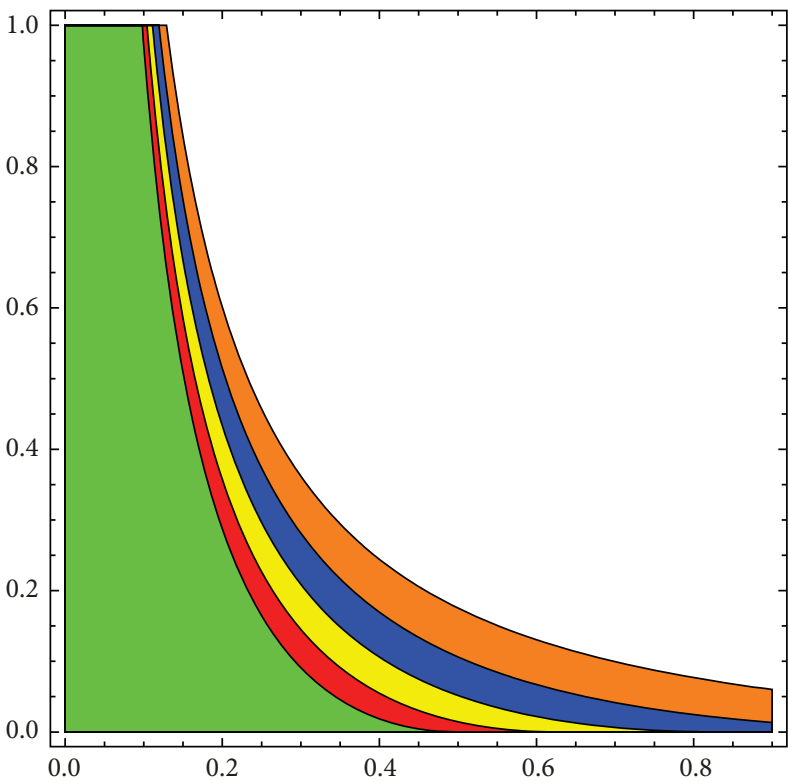

(a)

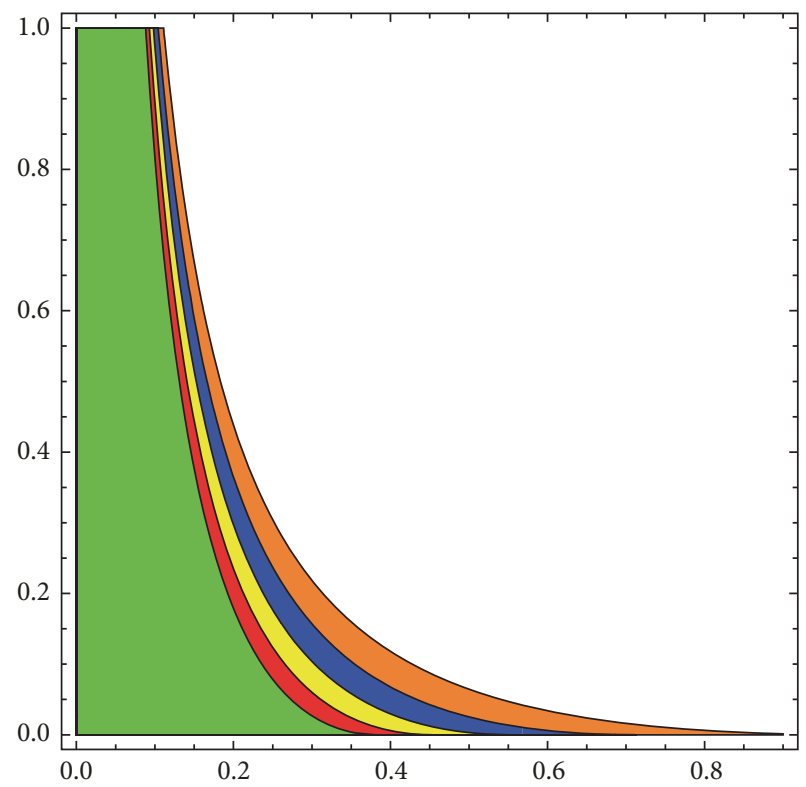

(b)

Figure 2: (a) Domain of parameters of (6) for $Q=0.4$. (b) Domain of parameters of (6) for $Q=1\left(K_{1}=0,0.2,0.4,0.6,0.8\right.$ represented by orange, blue, yellow, red, and green colors, resp.).

$$
\begin{aligned}
\| I & -A^{*-1}\left(F^{\prime}\left(\frac{x+y}{2}\right)+[x, y ; G]\right) \| \\
& \leq\left\|A^{*-1}\right\|\left\|A^{*}-F^{\prime}\left(\frac{x+y}{2}\right)-[x, y ; G]\right\| \\
& \leq \lambda\left\|F^{\prime}\left(\rho^{*}\right)+\left[\rho^{*}, \bar{\rho} ; G\right]-F^{\prime}\left(\frac{x+y}{2}\right)-[x, y ; G]\right\| \\
& \leq \lambda\left(\sigma_{1}\left(\left\|\frac{x+y}{2}-\rho^{*}\right\|\right)+\sigma_{2}\left(\left\|x-\rho^{*}\right\|,\|y-\bar{\rho}\|\right)\right) \\
& \leq \lambda\left(\sigma_{1}\left(R^{*}\right)+\sigma_{2}\left(R^{*}, R^{*}+\theta\right)\right)<1 .
\end{aligned}
$$

Using Banach lemma on invertible operators, it follows that $\left(F^{\prime}((x+y) / 2)+[x, y ; G]\right)^{-1}$ exists and

$$
\begin{aligned}
& \left\|\left(F^{\prime}\left(\frac{x+y}{2}\right)+[x, y ; G]\right)^{-1}\right\| \\
& \quad \leq \frac{\lambda}{1-\lambda\left(\sigma_{1}\left(R^{*}\right)+\sigma_{2}\left(R^{*}, R^{*}+\theta\right)\right)} .
\end{aligned}
$$

Theorem 7. If $\left(H, \rho^{*}, \bar{\rho}\right) \in U$ and Lemma 6 holds, then starting with $x_{0}, y_{0} \in \mathscr{B}\left(\rho^{*}, R^{*}\right)$, the sequences $\left\{x_{k}\right\}$ and $\left\{y_{k}\right\}$ generated by (6) are well defined and converge to the solution $\rho^{*}$ of (1). Moreover, the solution $\rho^{*}$ is unique in $\overline{\mathscr{B}}\left(\rho^{*}, R^{*}\right) \cap \mathscr{D}$. Furthermore, the following holds:

$$
\begin{aligned}
& \left\|x_{k+1}-x^{*}\right\| \leq \widetilde{P}\left\|x_{k}-x^{*}\right\|, \\
& \left\|y_{k+1}-x^{*}\right\| \leq \widetilde{P}\left\|x_{k+1}-x^{*}\right\|,
\end{aligned}
$$

where $\widetilde{P}=K \lambda /\left(1-\lambda\left(\sigma_{1}\left(R^{*}\right)+\sigma_{2}\left(R^{*}, R^{*}+\theta\right)\right)\right)$ and $K=$ $\int_{0}^{1} \sigma_{1}\left((1+u) R^{*}\right) d u+\sigma_{2}\left(R^{*}, 2 R^{*}\right)$.

Proof. Clearly, $x_{0}, y_{0} \in \mathscr{B}\left(\rho^{*}, R^{*}\right)$ and $A_{0}^{-1}$ exists from Lemma 6. Now,

$$
\begin{aligned}
\left\|x_{1}-\rho^{*}\right\| & \leq\left\|x_{0}-\rho^{*}-A_{0}^{-1}\left(H\left(x_{0}\right)-H\left(\rho^{*}\right)\right)\right\| \leq\left\|A_{0}^{-1}\right\|\left\|A_{0}\left(x_{0}-\rho^{*}\right)-\left(F\left(x_{0}\right)+G\left(x_{0}\right)-F\left(\rho^{*}\right)-G\left(\rho^{*}\right)\right)\right\| \\
& =\left\|A_{0}^{-1}\right\|\left\|F^{\prime}\left(\frac{x_{0}+y_{0}}{2}\right)+\left[x_{0}, y_{0} ; G\right]-\int_{0}^{1} F^{\prime}\left(\rho^{*}+u\left(x_{0}-\rho^{*}\right)\right) d u-\left[\rho^{*}, x_{0} ; G\right]\right\|\left\|x_{0}-\rho^{*}\right\| \\
& \leq \frac{\lambda\left(\int_{0}^{1} \sigma_{1}\left(\frac{\left\|x_{0}+y_{0}-2 \rho^{*}-2 u\left(x_{0}-\rho^{*}\right)\right\|}{2}\right) d u+\sigma_{2}\left(\left\|x_{0}-\rho^{*}\right\|,\left\|y_{0}-x_{0}\right\|\right)\right)}{1-\lambda\left(\sigma_{1}\left(R^{*}\right)+\sigma_{2}\left(R^{*}, R^{*}+\theta\right)\right)}\left\|x_{0}-\rho^{*}\right\| \\
& \leq \frac{\lambda\left(\int_{0}^{1} \sigma_{1}\left((1+u) R^{*}\right) d u+\sigma_{2}\left(R^{*}, 2 R^{*}\right)\right)}{1-\lambda\left(\sigma_{1}\left(R^{*}\right)+\sigma_{2}\left(R^{*}, R^{*}+\theta\right)\right)}\left\|x_{0}-\rho^{*}\right\|=\widetilde{P}\left\|x_{0}-\rho^{*}\right\| .
\end{aligned}
$$


From $U$, we get $\left\|x_{1}-\rho^{*}\right\|<\left\|x_{0}-\rho^{*}\right\|$ and $x_{1} \in \mathscr{B}\left(\rho^{*}, R^{*}\right)$.

Now,

$$
\begin{aligned}
\left\|y_{1}-\rho^{*}\right\| & =\left\|x_{1}-\rho^{*}-A_{0}^{-1}\left(H\left(x_{1}\right)-H\left(\rho^{*}\right)\right)\right\| \leq\left\|A_{0}^{-1}\right\|\left\|A_{0}\left(x_{1}-\rho^{*}\right)-\left(F\left(x_{1}\right)+G\left(x_{1}\right)-F\left(\rho^{*}\right)-G\left(\rho^{*}\right)\right)\right\| \\
& =\left\|A_{0}^{-1}\right\|\left\|F^{\prime}\left(\frac{x_{0}+y_{0}}{2}\right)+\left[x_{0}, y_{0} ; G\right]-\int_{0}^{1} F^{\prime}\left(\rho^{*}+u\left(x_{1}-\rho^{*}\right)\right) d u-\left[\rho^{*}, x_{1} ; G\right]\right\|\left\|x_{1}-\rho^{*}\right\| \\
& \leq \frac{\lambda\left(\int_{0}^{1} \sigma_{1}\left(\left\|x_{0}+y_{0}-2 \rho^{*}-2 u\left(x_{1}-\rho^{*}\right)\right\| / 2\right) d u+\sigma_{2}\left(\left\|x_{0}-\rho^{*}\right\|,\left\|x_{1}-y_{0}\right\|\right)\right)}{1-\lambda\left(\sigma_{1}\left(R^{*}\right)+\sigma_{2}\left(R^{*}, R^{*}+\theta\right)\right)}\left\|x_{1}-\rho^{*}\right\| \\
& \leq \frac{\lambda\left(\int_{0}^{1} \sigma_{1}\left((1+u) R^{*}\right) d u+\sigma_{2}\left(R^{*}, 2 R^{*}\right)\right)}{1-\lambda\left(\sigma_{1}\left(R^{*}\right)+\sigma_{2}\left(R^{*}, R^{*}+\theta\right)\right)}\left\|x_{0}-\rho^{*}\right\|=\widetilde{P}\left\|x_{1}-\rho^{*}\right\| .
\end{aligned}
$$

Thus, $\left\|y_{1}-\rho^{*}\right\|<\left\|x_{1}-\rho^{*}\right\|$ and $y_{1} \in \mathscr{B}\left(\rho^{*}, R^{*}\right)$. Proceeding in a similar way, it follows that $\left\|y_{k}-\rho^{*}\right\|<\left\|x_{k}-\rho^{*}\right\|<\left\|x_{k-1}-\rho^{*}\right\|$ for each $k \in \mathbb{N}$. This gives $\lim _{k \rightarrow \infty} x_{k}=\rho^{*}$ and consequently $y_{k} \rightarrow \rho^{*}$ as $k \rightarrow \infty$. It remains to show the uniqueness of $\rho^{*}$. It can be proved in a similar manner as proved in Theorem 4 . This proves the theorem.

3.1. On the Accessibility and Some Special Cases. In this section, we present some special cases of Theorem 7 and (6). We establish the region of accessibility for $\rho^{*}$. A solution $\rho^{*}$ is said to be accessible from those points $x_{0}$ and $y_{0}$ if the sequences $\left\{x_{k}\right\}$ and $\left\{y_{k}\right\}$ given by (6) converge to $\rho^{*}$. The set of a combination of all such points for which the sequences $\left\{x_{k}\right\}$ and $\left\{y_{k}\right\}$ converge to $\rho^{*}$ is called the region of accessibility of $\rho^{*}$. We use here Theorem 7 to find the region of accessibility of (6). We consider here $F=0$ and replace the condition $\left(L_{2}\right)$ by

$$
\|[x, y ; G]-[u, v ; G]\| \leq K_{1}+K_{2}(\|x-u\|+\|y-v\|) .
$$

It is indicated in Section 2.1 that this type of condition arises for nondifferentiable operators. Now, we present the local convergence analysis of (6) using condition (38). If condition (38) is used, then

$$
R^{*}=\frac{1-2 \lambda K_{1}-\lambda K_{2} \theta}{5 \lambda K_{2}} .
$$

$R^{*}$ should be the real positive number which is possible only when

$$
1-2 \lambda K_{1}-\lambda K_{2} \theta>0 .
$$

To verify condition (40), we draw the region by taking $\lambda K_{1}=$ $y$ and $\lambda K_{2}=x$ and then taking different values of $\theta$ to see the difference between the convergence regions. This can be seen by Figure 3. Now, using (40), it is observed that the condition $\left(L_{5}\right)$ is equivalent to the condition for such $R^{*}$, which is

$$
0<1-\lambda K_{1}-\lambda K_{2} \theta .
$$

Obviously, the condition satisfying (40) satisfies (41). This can be seen in Figure 4. We come to the conclusion that, with a smaller distance between $\rho^{*}$ and $\bar{\rho}$, a larger domain is achieved.

Let us discuss the local convergence of (6) which does not use the differentiability condition.

Theorem 8. Let $G: X \rightarrow Y$ be a nonlinear operator. Suppose that

$\left(D_{1}\right)$ there exists $\rho^{*}$ such that $G\left(\rho^{*}\right)=0$; choose $\bar{\rho} \in \mathscr{D}$ so that $\left\|\rho^{*}-\bar{\rho}\right\|=\theta>0$ so that the operator $\left[\rho^{*}, \bar{\rho} ; G\right]$ is invertible and $\left\|\left[\rho^{*}, \bar{\rho} ; G\right]^{-1}\right\| \leq \lambda$;

$\left(D_{2}\right)\|[x, y ; G]-[u, v ; G]\| \leq K_{1}+K_{2}(\|x-u\|+\|y-v\|)$;

$\left(D_{3}\right) 1-2 \lambda K_{1}-\lambda K_{2} \theta>0$.

Then, starting with $x_{0}, y_{0} \in \mathscr{B}\left(\rho^{*}, R^{*}\right)$, the sequences $\left\{x_{k}\right\}$ and $\left\{y_{k}\right\}$ given by (6) are well defined and converge to the unique solution $\rho^{*}$ of (4), where $R^{*}=\left(1-2 \lambda K_{1}-\lambda K_{2} \theta\right) / 5 \lambda K_{2}$.

Proof. The proof follows the same lines as the theorems given above. So, we omit the proof here.

\section{Numerical Experiments}

In this section, some numerical examples are given to demonstrate the applicability and efficacy of our work.

Example 9. Consider the nonlinear system

$$
\begin{gathered}
u^{3 / 2}-v-\frac{3}{4}+0.005|u-1|=0 \\
v^{3 / 2}+\frac{2}{9} u-\frac{3}{8}+0.005|u|=0
\end{gathered}
$$

for $x=(u, v) \in \mathbb{R}^{2}$.

Consider the operator $H: \mathscr{D} \subset \mathbb{R}^{2} \rightarrow \mathbb{R}^{2}$, where $\mathscr{D}=$ $\left\{(u, v) \in \mathbb{R}^{2} ; u, v>0\right\}$. We take $H(x)=F(x)+G(x)$, where $F=\left(F_{1}, F_{2}\right)$ and $G=\left(G_{1}, G_{2}\right)$ such that $F_{1}(u, v)=u^{3 / 2}$ $v-=3 / 4, F_{2}(u, v)=v^{3 / 2}+(2 / 9) u-3 / 8, G_{1}(u, v)=0.005 \mid u-$ $1 \mid$, and $G_{2}(u, v)=0.005|v|$. Here, we take the norm $\|x\|_{\infty}=$ $\max _{1 \leq i \leq 2}\left|x_{i}\right|$ for vectors and the corresponding norm $\|A\|=$ 


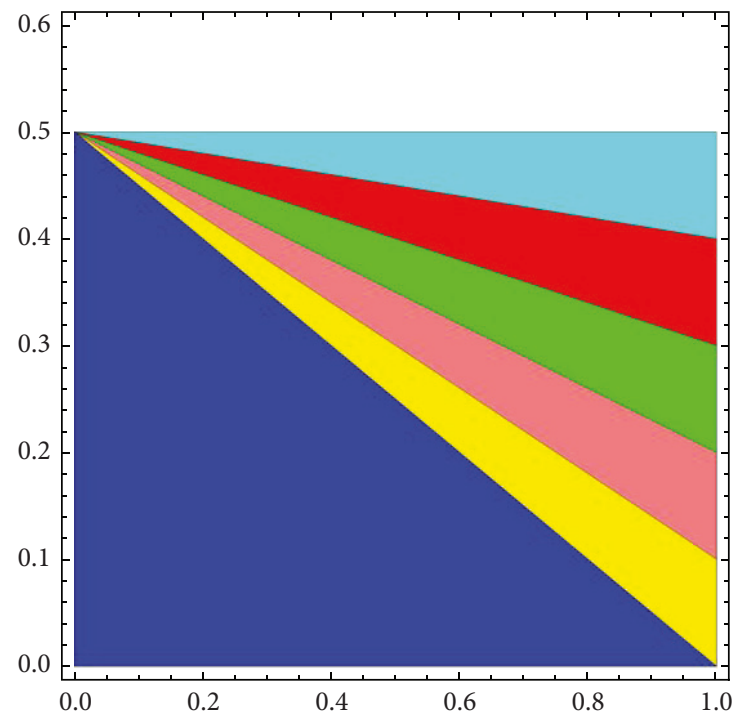

Figure 3: Region of $x y$ plane where (40) holds true for $\theta=$ $0,0.2,0.4,0.4,0.6,0.8,1$ represented by cyan, red, green, pink, yellow, and blue colors, respectively.

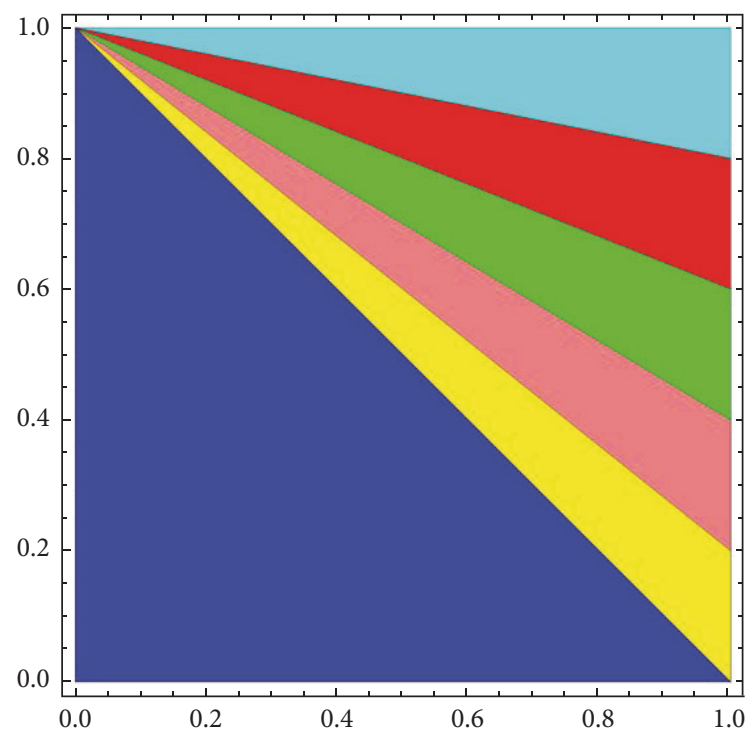

FIgURE 4: Region of $x y$ plane where (41) holds true for $\theta=$ $0,0.2,0.4,0.4,0.6,0.8,1$ represented by cyan, red, green, pink, yellow, and blue colors, respectively.

$\max _{1 \leq j \leq 2} \sum_{k=1}^{2}\left|a_{j k}\right|$ for the matrix $A \in \mathbb{R}^{2} \times \mathbb{R}^{2}$. Now, by the definition of divided difference for $1 \leq i, j \leq n$,

$$
\begin{aligned}
& {[s, t ; G]_{i j}=\frac{1}{s_{j}-t_{j}}\left(G_{i}\left(s_{1}, \ldots, s_{j-1}, s_{j}, t_{j+1}, \ldots, t_{n}\right)\right.} \\
& \left.-G_{i}\left(s_{1}, \ldots, s_{j-1}, t_{j}, t_{j+1}, \ldots, t_{n}\right)\right),
\end{aligned}
$$

for $s, t \in \mathbb{R}^{n}$. We get

$$
\|[x, y ; G]-[u, v ; G]\| \leq \frac{1}{100},
$$

TABLE 2: Absolute error obtained by (6).

\begin{tabular}{lc}
\hline$k$ & $\left\|\rho^{*}-x_{k}\right\|$ \\
\hline 1 & $1.7275 \ldots \times 10^{-03}$ \\
2 & $7.5607 \ldots \times 10^{-08}$ \\
3 & $2.7441 \ldots \times 10^{-17}$ \\
\hline
\end{tabular}

$$
\left\|F^{\prime}(x)-F^{\prime}(y)\right\| \leq \frac{3}{2}\|x-y\|^{1 / 2}
$$

We get $\omega_{1}(t)=(3 / 2) t^{1 / 2} \beta$ and $\omega_{2}(r, s)=(1 / 100) \beta$. We take the starting points $x_{0}=(0.9638,0.2376)$ and $y_{0}=$ $(0.9629,0.2382)$. Now, using Theorem 4 , we can easily obtain that $\alpha=0.0009, \beta=1.3327, \eta=0.05693226, M=$ $0.3348821, R=0.148404722$, and $\vec{M}=0.643087952974343$. It can be easily seen that $M+\widetilde{M}=0.97797<1$. So, all conditions of Theorem 4 are true and hence (6) can be applied to Example 9. After 4 iterations, we get that the iteration converges to the solution $\rho^{*}=(1.0207 \ldots, 0.27988 \ldots)$. Absolute error $\left(\left\|\rho^{*}-x_{k}\right\|\right)$ is given in Table 2 with tolerance $10^{-20}$.

Example 10 (see [18]). Consider

$$
x(t)=f(t)+\int_{a}^{b} G_{1}(t, s) H_{1}(s, x(s)) d s,
$$

$$
t \in[a, b],
$$

where $a, b \in(-\infty, \infty), f, H_{1}, G_{1}$ are given functions and $x$ is the solution of (45), to be determined. This problem can be transformed to solve $H(x)=0$, where $H: \mathscr{D} \subset C[a, b] \rightarrow$ $C[a, b]$, given by

$$
\begin{aligned}
{[H(x)](t)=} & x(t)-f(t) \\
& +\int_{a}^{b} G_{1}(t, s) H_{1}(s, x(s)) d s,
\end{aligned}
$$

$$
t \in[a, b] .
$$

Consider $G_{1}(t, s)$ as a green function in $[a, b] \times[a, b]$, and we use a discretization process to transform (46) into a finite dimensional problem. For this, we implement the GaussLegendre formula, given by

$$
\int_{a}^{b} Q(s) d s=\sum_{j=1}^{l} w_{j} Q\left(s_{j}\right),
$$

where $l$ is the number of nodes. $w_{j}, s_{j}$ are weights and nodes, respectively, to be determined. We denote the approximation of $x\left(s_{j}\right)$ and $f\left(s_{j}\right)$ by $x_{j}$ and $f_{j}$, respectively, for $j=1, \ldots, l$. Then, (46) is equivalent to solving a system of nonlinear equations, given by

$$
H_{j}=x_{j}-f_{j}-\sum_{k=1}^{l} a_{j, k} H_{1}\left(s_{k}, x_{k}\right), \quad j=1, \ldots, l,
$$


TABLE 3: Approximation of solution of (52).

\begin{tabular}{lc}
\hline$j$ & $\rho^{*}$ \\
\hline 1 & $0.5064627 \ldots$ \\
2 & $0.5307228 \ldots$ \\
3 & 0.5617389 \\
4 & 0.58321990 \\
5 & $0.5832199 \ldots$ \\
6 & $0.5617389 \ldots$ \\
7 & $0.5307229 \ldots$ \\
8 & $0.5064628 \ldots$ \\
\hline
\end{tabular}

where

$$
\begin{aligned}
a_{j, k} & =w_{j} G_{1}\left(s_{j}, s_{k}\right) \\
& = \begin{cases}w_{j} \frac{\left(b-s_{j}\right)\left(s_{k}-a\right)}{b-a} & \text { if } k \leq j, \\
w_{k} \frac{\left(b-s_{k}\right)\left(s_{j}-a\right)}{b-a} & \text { if } j>k .\end{cases}
\end{aligned}
$$

With this transformation, (48) is equivalent to

$$
H(x) \equiv x-f-A z=0, \quad H: \mathbb{R}^{l} \longrightarrow \mathbb{R}^{l},
$$

$x=\left(x_{1}, \ldots, x_{l}\right)^{T}, f=\left(f_{1}, \ldots, f_{l}\right)^{T}, A=\left(a_{j, k}\right)_{j, k=1}^{l}$, and $z=$ $\left(H_{1}\left(s_{1}, x_{1}\right), \ldots, H_{l}\left(s_{l}, x_{l}\right)\right)$. Now, we consider the nonlinear integral equation

$$
\begin{aligned}
& x(s)=\frac{1}{2}+\frac{3}{4} \int_{0}^{1} G_{1}(t, s)\left(x(s)^{2}+|x(s)|\right) d s, \\
& \\
& s \in[0,1] ;
\end{aligned}
$$

using the transformation defined above, by taking $l=8$, this equation can be transformed to a nonlinear system of equations:

$$
H(x)=x-\frac{1}{2}-\frac{3}{4} A\left(u_{x}+v_{x}\right)
$$

where $x=\left(x_{1}, \ldots, x_{8}\right)^{T}, \mathbf{1} / \mathbf{2}=(1 / 2, \ldots, 1 / 2)^{T}, u_{x}=$ $\left(x_{1}^{2}, \ldots, x_{8}^{2}\right)^{T}$, and $v_{x}=\left(\left|x_{1}\right|, \ldots,\left|x_{8}\right|\right)^{T}$. Choosing $x_{0}=$ $(2 / 5, \ldots, 4 / 5)^{T}, y_{0}=(1 / 2, \ldots, 1 / 2)^{T}$ and with the help of (21) and Theorem 5 , we get $K_{1}=0.185338488109776, K_{2}=$ $0.092669244054888, \beta=1.05121302729, \eta=0.1818592275$, $\alpha=0.1, T_{1}=0.222287582674836$, and $T_{2}=0.23026190887$. From (22), we get $R=0.260442310241258$. All the conditions of Theorem 5 are true as $T_{1}+T_{3}(R)=0.4776013635212<1$ and $T_{2}+T_{3}(R)=0.48557568972059<1$. So, we can ensure the semilocal convergence of (6). After 3 iterations, we get the approximate solution, which is given in Table 3 with tolerance $10^{-15}$. An approximate error to the solution is given in Table 4 .

Example 11. Turning to Example 1, it can be easily seen that $\rho^{*}=(1,0.25)$. Now, to apply Theorem 7 , we take $\bar{\rho}=(1.5,0.5)$, and then $\theta=0.5$ and $\lambda=1.1562799 \ldots$. We consider $\rho_{1}(t)=(3 / 2) t^{1 / 2}$ and $\rho_{2}(s, t)=2 / 9$. Also,
TABLE 4: Absolute error obtained by (6).

\begin{tabular}{lc}
\hline$j$ & $\left\|\rho^{*}-x_{j}\right\|$ \\
\hline 1 & $1.3606742 \times 10^{-03}$ \\
2 & $3.5262214 \times 10^{-09}$ \\
3 & $1.1814916 \times 10^{-16}$ \\
\hline
\end{tabular}

there exists $R^{*}=0.015953030586386$ that verifies (32) and $\lambda\left(\rho_{1}\left(R^{*}\right)+\rho_{2}\left(R^{*}, R^{*}+\theta\right)\right)=1.1562799(0.18945796+$ $2 / 9)=0.476017545958554<1$. Thus, all the conditions of Theorem 7 hold true and thus the ball of convergence along with its domain of uniqueness of ball is given by $\left\{x \in \mathbb{R}^{2}\right.$ : $\left.\left\|x-\rho^{*}\right\| \leq 0.015953\right\}$.

\section{Conclusions}

In this paper, we established a new convergence analysis of the two-step iterative method for solving nonlinear nondifferentiable equations. Using some recurrences, we analyze the semilocal as well as local convergence analysis for this method. The novelty of this work lies in the fact that it avoids the differentiability condition in the convergence analysis for nondifferentiable operators, a contrast with earlier studies to take into account. This is very important for the practical purpose. In semilocal convergence analysis, theorems are given for existence-uniqueness balls. Moreover, the domain of the parameters is given to show the guaranteed convergence of the method and suitability of the starting points. In local convergence analysis, we avoid the differentiability condition on the involved operator as a contrast to the earlier study. This way, the applicability of local convergence theorem is extended. Theorems are provided for the existenceuniqueness ball. Furthermore, its region of accessibility is given and an idea for enlarging the convergence domain is provided. Finally, some numerical examples including nonlinear Hammerstein type integral equations are given to validate the theoretical results obtained by us.

\section{Conflicts of Interest}

The authors declare that there are no conflicts of interest regarding the publication of this paper.

\section{Acknowledgments}

This research was supported in part by the project of Generalitat Valenciana Prometeo/2016/089 and MTM2014-52016C2-2-P of the Spanish Ministry of Science and Innovation.

\section{References}

[1] H. Ren and I. K. Argyros, "On the convergence of KingWerner-type methods of order 1+2 free of derivatives," Applied Mathematics and Computation, vol. 256, pp. 148-159, 2015.

[2] J. A. Ezquerro, M. A. Hernández-Verón, and A. I. Velasco, "An analysis of the semilocal convergence for Secant-like methods," Applied Mathematics and Computation, vol. 266, Article ID 21281, pp. 883-892, 2015. 
[3] P. K. Parida and D. K. Gupta, "Semilocal convergence of a family of third-order Chebyshev-type methods under a mild differentiability condition," International Journal of Computer Mathematics, vol. 87, no. 15, pp. 3405-3419, 2010.

[4] L. B. Rall, Computational Solution of Nonlinear Operator Equations, John Wiley \& Sons, New York, NY, USA, 1969.

[5] L. V. Kantorovich and G. P. Akilov, Functional Analysis in Normed Spaces, International Series of Monographs in Pure and Applied Mathematics, 1964.

[6] J. F. Traub, Iterative Methods for the Solution of Equations, American Mathematical Society, 1982.

[7] M. Prashanth and D. K. Gupta, "Semilocal convergence for Super-Halley's method under $\omega$-differentiability condition," Japan Journal of Industrial and Applied Mathematics, vol. 32, no. 1, pp. 77-94, 2015.

[8] M. A. Hernández-Verón and M. J. Rubio, "On the ball of convergence of Secant-like methods for non-differentiable operators," Applied Mathematics and Computation, vol. 273, pp. 506-512, 2016.

[9] I. K. Argyros, Á. A. Magreñán, L. Orcos, and J. A. Sicilia, "Local convergence of a relaxed two-step Newton like method with applications," Journal of Mathematical Chemistry, vol. 55, no. 7, pp. 1427-1442, 2017.

[10] R. Behl, A. Cordero, S. S. Motsa, and J. R. Torregrosa, "On developing fourth-order optimal families of methods for multiple roots and their dynamics," Applied Mathematics and Computation, vol. 265, pp. 520-532, 2015.

[11] T. Yamamoto, "Historical developments in convergence analysis for Newton's and Newton-like methods," Journal of Computational and Applied Mathematics, vol. 124, no. 1-2, pp. 1-23, 2000.

[12] I. K. Argyros, R. Behl, and S. S. Motsa, "Unifying semilocal and local convergence of Newton's method on Banach space with a convergence structure," Applied Numerical Mathematics, vol. 115, pp. 225-234, 2017.

[13] J. M. Gutierrez and M. A. Hernandez, "A family of ChebyshevHalley type methods in Banach spaces," Bulletin of the Australian Mathematical Society, vol. 55, no. 1, pp. 113-130, 1997.

[14] M. A. Hernández-Verón and M. J. Rubio, "On a NewtonKurchatov-type iterative process," Numerical Functional Analysis and Optimization, vol. 37, no. 1, pp. 65-79, 2016.

[15] S. M. Shakhno, "Convergence of the two-step combined method and uniqueness of the solution of nonlinear operator equations," Journal of Computational and Applied Mathematics, vol. 261, pp. 378-386, 2014.

[16] A. Kumar, D. K. Gupta, E. Martnez, and S. Singh, "Semilocal convergence of a secant-type method under weak Lipschitz conditions in Banach spaces," Journal of Computational and Applied Mathematics, vol. 330, pp. 732-741, 2018.

[17] I. K. Argyros and H. Ren, "On the convergence of efficient KingWerner-type methods of order 1+2," Journal of Computational and Applied Mathematics, vol. 285, pp. 169-180, 2015.

[18] D. D. Bruns and J. E. Bailey, "Nonlinear feedback control for operating a nonisothermal CSTR near an unstable steady state," Chemical Engineering Science, vol. 32, no. 3, pp. 257-264, 1977. 


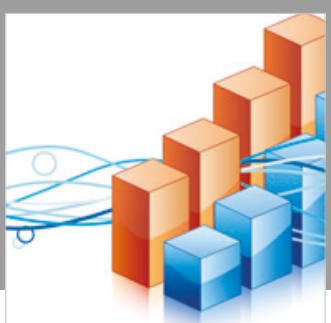

Advances in

Operations Research

\section{-n-m}
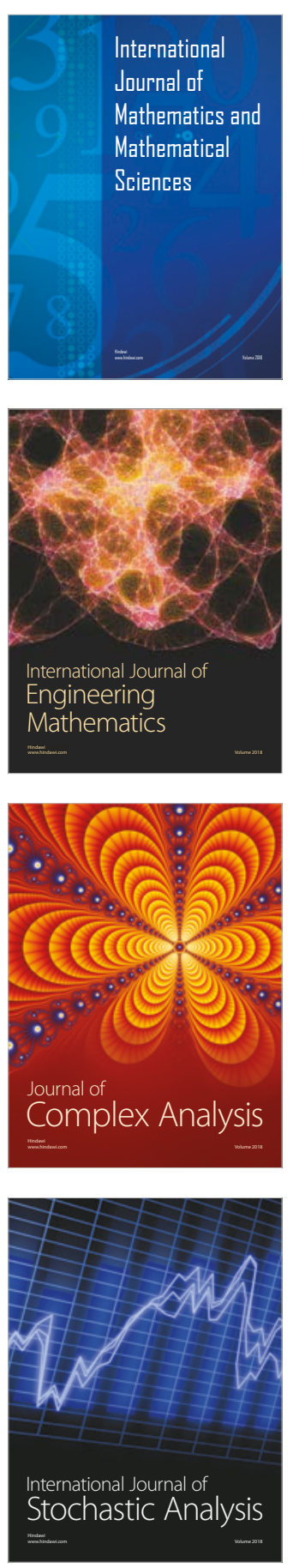
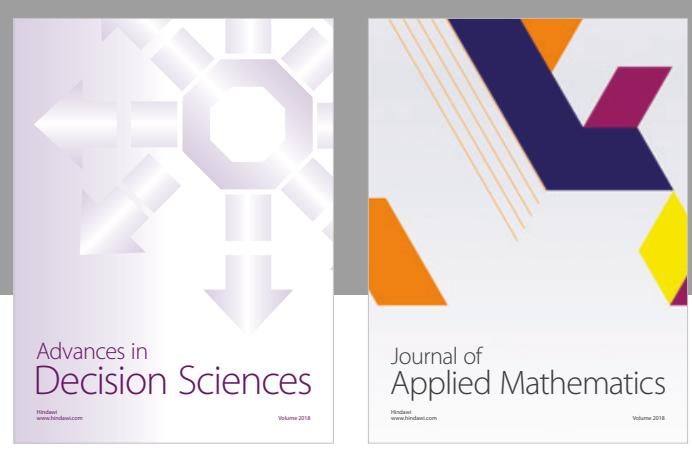

Journal of

Applied Mathematics
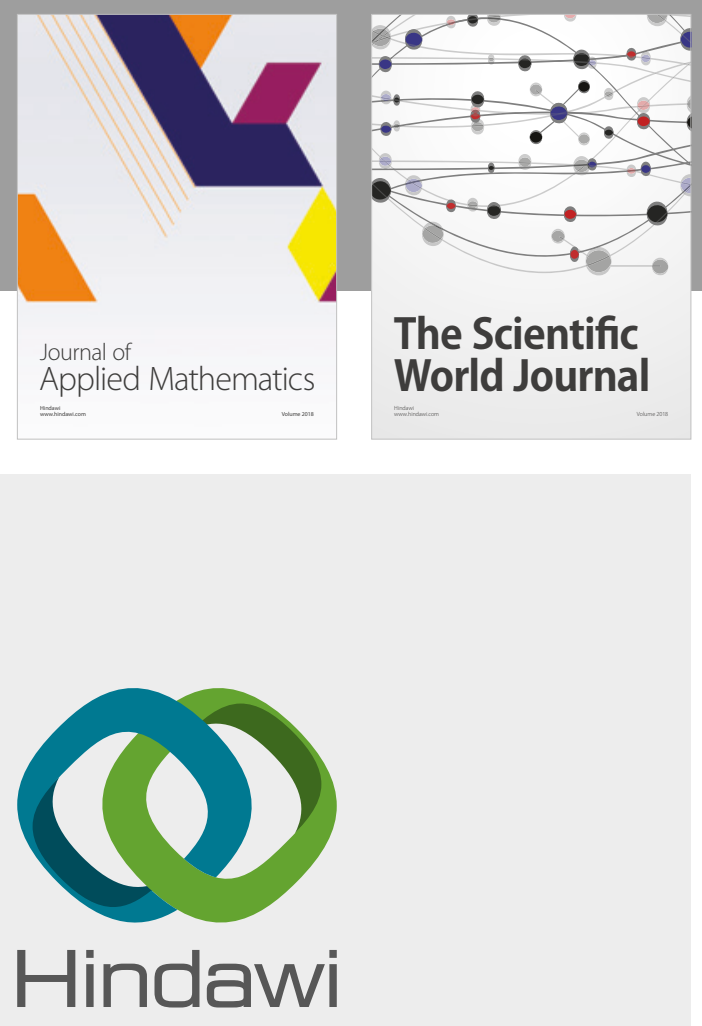

Submit your manuscripts at

www.hindawi.com

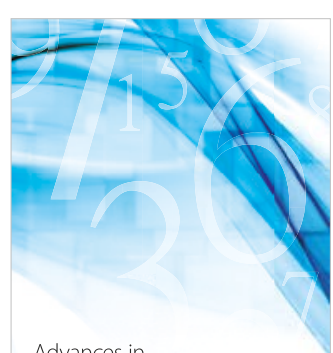

Advances in
Numerical Analysis
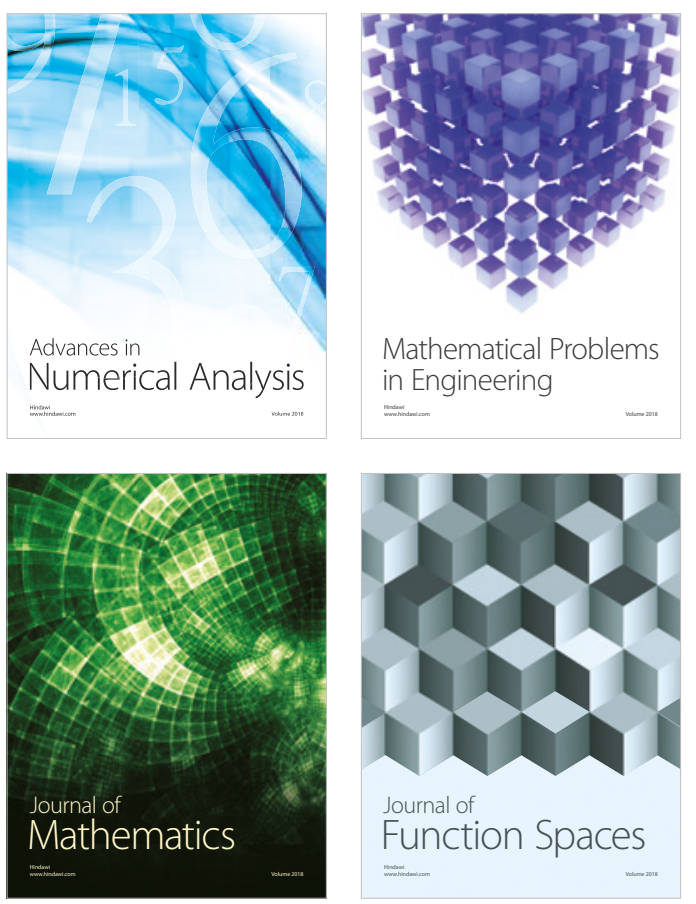

Mathematical Problems in Engineering

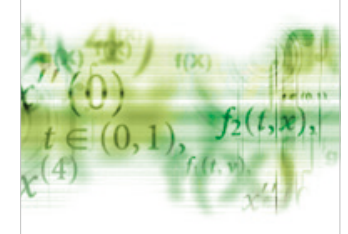

International Journal of

Differential Equations

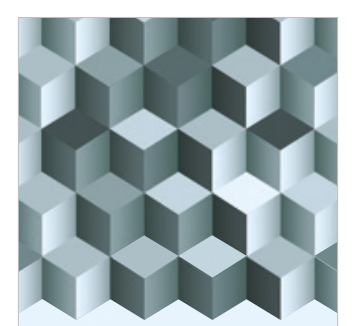

Journal of

Function Spaces
The Scientific

World Journal

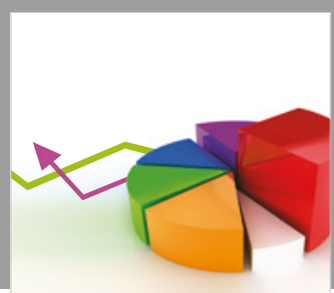

Journal of

Probability and Statistics
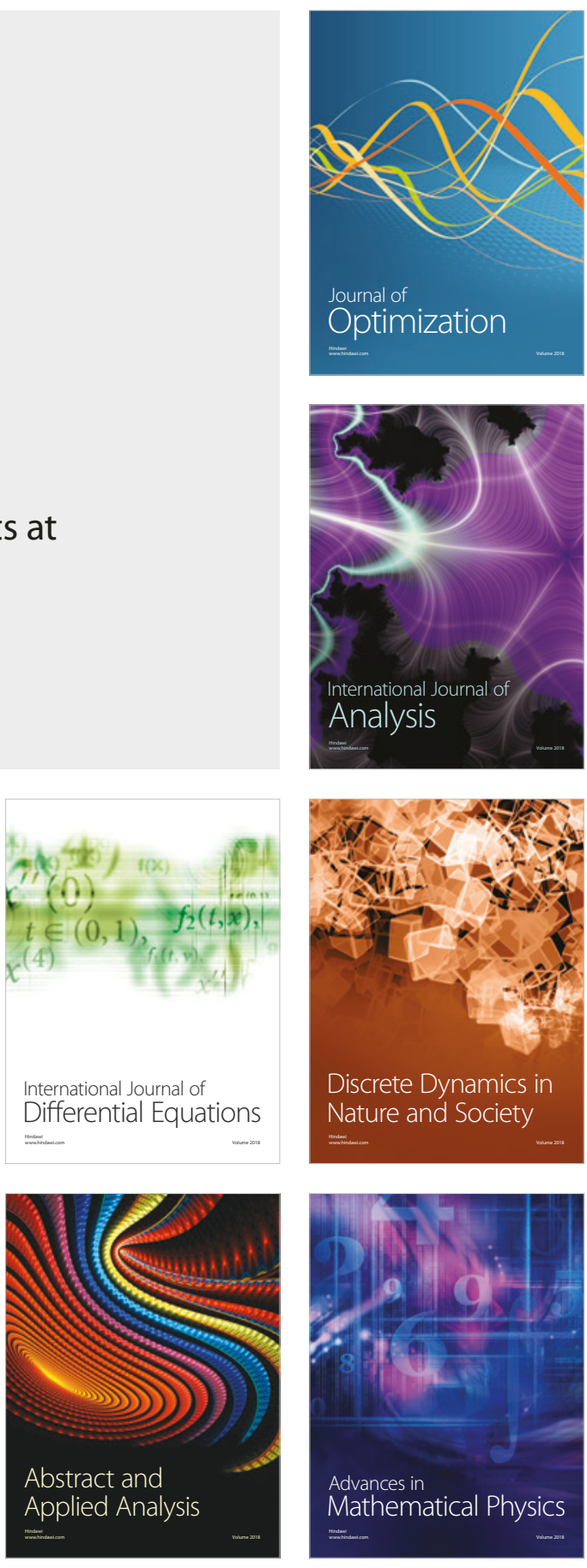